\title{
A TEST OF THE GREAT CIRCLE APPROXIMATION IN THE ANALYSIS OF SURFACE WAVES
}

\author{
Dolors Alsina and Roel Snieder
}

Department of Theoretical Geophysics, Utrecht University

Valerie Maupin

Institute de Physique du Globe de Strasbourg

\begin{abstract}
A new way of reconstructing wave fronts is developed for testing the validity of the great circle approximation in dispersion analysis of surface waves. Phase and time delays between pairs of stations of an array are used to reconstruct the wave fronts or surfaces of equal phase. This technique is applied to fundamental mode Rayleigh waves recorded at the NARS stations during the IIIHA (Iberian Lithosphere Heterogeneity and Anisotropy) project. The resulting wave fronts are compared with the theoretical ones, i.e. calculated assuming that the waves have traveled along the great circle path with a constant phase velocity, and the angle between both fronts is calculated. Waves traveling mostly through a laterally homogeneous oceanic path before arriving to the Iberian Peninsula are analyzed for the frequency range of 10 to $60 \mathrm{mHz}$. These show angles up to 8 degrees between the theoretical wave fronts and the wave fronts reconstructed using the delay data. This implies that we make a relative error less than $1 \%$ when calculating pathaveraged phase velocities ignoring the deviation of the arrival of the wave from the great circle direction.
\end{abstract}

\section{Introduction}

One of the goals of modern seismology is to map the lateral heterogeneity in the Earth. The analysis of surface wave data is particularly valuable for studying lateral variations of the crust and upper mantle. Standard surface wave tomography relies on the great circle approximation which states that surface waves are only influenced by the integral of the local phase or group velocity over the source-receiver minor arc. However, the great circle approximation cannot be used if lateral variations have produced strong deviations of the surface wave ray path and if the structure is not smooth on a scale of a wavelength.

The goals of this research are (1) to test whether the observed wavefield arrives in the great circle direction, and (2) to evaluate the bias in phase velocity calculations in case a deviation is observed. For this purpose a new way of calculating wave fronts is developed. This is applied to data from the NARS network, recorded during the ILIHA project. An array enables the measurement of the azimuth of the direction of arrival of the wave. The phase velocity $c_{\text {meas }}$ measured along the great circle can be related to the real local one $c_{\text {real }}$ by the expression $c_{\text {meas }}=c_{\text {real }} / \cos \alpha, \alpha$ being the deviation of the ray path from the great circle direction. Phase delays for the fundamental mode of the Rayleigh waves are calculated between the stations for different frequency bands. The wave fronts are represented by lines connecting equal phase delays. The angle $\alpha$ between the observed and the theoretical wave fronts (corresponding to waves traveling through a homogeneous medium) is calculated. This angle provides information about the deviation of the path of the true wave from the source-receiver minor arc; it can thus be

Copyright 1993 by the American Geophysical Union.

Paper number 93GL00774

0094-8534/93/93GL-00774\$03.00 used as a criterion of validity of the great circle approximation and as an indication of ray bending effects. Earlier works have already been done to compute phase velocities and the approach azimuth of the wave train for a given period, by means of a triangular network [Evernden, 1954]. Although the tripartite phase velocity method was designed to handle surface waves incident upon the array from any azimuth, the results of Knopoff et al.[1967] showed that when the direction of propagation does not lie along one of the legs of the network, the phase velocities computed may be in significant error. Schwab and Kausel [1976] proposed the extension to quadripartite networks to solve this limitation. With only 3 stations of a tripartite array one must assume plane wave fronts; in order to allow for curvature of the wavefronts, a fourth station is included in the array. In the 4 station's method, the wave front is approximated by the arc of a circle diverging from, or converging toward a fixed point on the free surface. In the method proposed in this paper, the form of the reconstructed wave front is not fixed a priori. The difference in curvature between the reconstructed and the theoretical wave fronts gives us information about lateral heterogeneities in the lithosphere and upper mantle both below the array and along the whole path along which the wave has traveled.

\section{Reconstruction of wavefronts: method}

In the geometrical optics approximation the wavefield can be expressed as $u(r, \omega)=A(r, \omega) e^{i \omega \tau(r, \omega)}$ in the frequency domain. Wave fronts are surfaces of equal phase $\phi=\omega \times \tau(r, \omega)$. The Eikonal equation $|\nabla \tau|^{2}=1 / \mathrm{c}^{2}$ determines the wave fronts, since it gives a constraint on the function $\tau(r, \omega)$. In the right-hand side of the Eikonal equation for dispersive surface waves, $\mathrm{c}$ is the local phase velocity.

To reconstruct the wave fronts we first measure the phases of the fundamental Rayleigh mode as a function of the frequency. The phases are measured using the residual dispersion analysis method described by Dziewonski et al. [1972]. The travel time function for each station is calculated by dividing the measured phases by $\omega$. For each event the station with the shortest epicentral distance is taken as a reference, and the difference between its travel time and the travel time of the other available stations is calculated. Afterwards the time delays are averaged over frequency bands of 6 or $8 \mathrm{mHz}$, between 10 and $60 \mathrm{mHz}$.

Next we perform weighted fitting of the phase plane by both forcing the delay time function to satisfy the delay time data while being as close as possible to a solution of the Eikonal equation. Our null hypothesis is that waves come in along the great circle and travel through the region of study with a fixed phase velocity $c_{i n}$. From all the functions $\tau(r)$ that fit the measured delay time data, we want to find the one closest to our initial hypothesis, i.e. satisfying the Eikonal equation $|\nabla \tau|^{2}=1 / c_{\text {in }}^{2}$, with $c_{\text {in }}$ the phase velocity corresponding to a realistic laterally homogeneous model. We apply thus a regularization based on the Eikonal equation: this criterion allows the reconstructed wave fronts to deviate from the ones in the laterally homogeneous reference model only when this is required by the data. The Mercator transformation is used

$$
y=\ln (\tan (\Theta / 2)), \quad x=\phi
$$


with $\Theta$ and $\phi$ the colatitude and longitude. An optimum fit is achieved by minimizing the sum:

$$
\mathrm{S}=\frac{\varepsilon}{\gamma} \sum_{\mathrm{i}}\left(\mathrm{d}_{\mathrm{i}}-\tau\left(\mathrm{r}_{\mathrm{i}}\right)\right)^{2}+\frac{(1-\varepsilon)}{\beta} \int\left[|\nabla \tau|^{2}-\frac{1}{\mathrm{c}_{\mathrm{in}}^{2}}\right]^{2} \mathrm{dxdy}
$$

where $d_{i}$ represents the delay time data, $\tau\left(\mathbf{r}_{i}\right)$ the delay time after inversion and $c_{\text {in }}$ the input phase velocity or the theoretical phase velocity we assume for a homogeneous structure of the region of study; $\gamma$ and $\beta$ are scaling factors such that both terms in the expression (2) are of order unity. All the stations have the same weight. As initial phase velocity we used values determined by Payo [1970] in the Iberian shield by means of a Long Period Triangular Array. The second term incorporates all our a priori notions about the solution into $\tau\left(r_{i}\right) .0 \leq \varepsilon \leq 1$ weights the relative importance of the two terms: if $\varepsilon$ is close to 0 , the phase function is a solution of the Eikonal equation but with a relatively bad fit to the data, and vice versa if $\varepsilon$ is close to 1 . As (2) is non-linear in $\tau\left(\mathbf{r}_{\mathrm{i}}\right)$ an iterative approach is needed. The minimization is performed by using a conjugate gradients method. The summation in the function $S$ is over the pairs of stations. The gradient of the phase function is parameterized as follows:

$$
\begin{aligned}
& \partial_{x} \tau=\sum_{n m} a_{n m} T_{n}(x) T_{m}(y) \\
& \partial_{y} \tau=\sum_{n m} b_{n m} T_{n}(x) T_{m}(y)
\end{aligned} n=0, N, m=0, \ldots, M(3)
$$

where $T_{n}(x)$ represents the Chebyschev polynomial of order $n$ and direction $x$. The condition

$$
\partial_{y}\left(\partial_{x} \tau\right)=\partial_{x}\left(\partial_{y} \tau\right)
$$

links the coefficients $a_{n m}$ and the $b_{n m}$, so that the coefficients $b_{n m}$ with $n>0$ can be expressed in the $a_{n m}$.

The wave fronts are represented by lines connecting equal time delays. Note that in the 2 nd term of the function $S$, the regularization term, there is no information about the direction of the gradient of the phase, but only about its norm. In other words, with this method we can obtain information about the direction of propagation of the waves without an a priori assumption for this direction. The area comprised between $36^{\circ} \mathrm{N}$ and $44^{\circ} \mathrm{N}$ and between $8^{\circ} \mathrm{W}$ and $2^{\circ} \mathrm{E}$ is the area represented in figure 2 and 3 . However, the inversion is only performed in the region covered by the stations from which the data are used. We calculate the angle $\alpha_{i}\left(x_{i}, y_{i}\right)$ between the theoretical wave fronts (i.e. computed assuming the waves had traveled through a homogeneous medium with a phase velocity $c_{\text {in }}$ and the experimental wave fronts (i.e. calculated using phase differences). For this we use the computed phase gradient vectors for both wavefields. The values obtained in the region covered by the stations, are averaged to give a mean value of the angle:

$$
\tilde{\alpha}=\frac{1}{\mathrm{n}} \sum_{\mathrm{i}=1}^{\mathrm{n}} \alpha_{\mathrm{i}}\left(\mathrm{x}_{\mathrm{i}}, \mathrm{y}_{\mathrm{i}}\right)
$$

where $n$ is the number of grid points $\left(x_{i}, y_{i}\right)$ used for the inversion. In our inversion a grid of $20 \times 20$ points has been used. By definition, a clockwise rotation of the experimental wave fronts with respect to the theoretical ones gives negative angles, while an anticlockwise rotation implies positive values. The standard deviation of the values is calculated as

$$
\sigma_{\alpha}=\left(\frac{1}{\mathrm{n}} \sum_{\mathrm{i}=1}^{\mathrm{n}}\left(\alpha_{\mathrm{i}}\left(\mathrm{x}_{\mathrm{i}}, \mathrm{y}_{\mathrm{i}}\right)-\bar{\alpha}\right)^{2}\right)^{\frac{1}{2}}
$$

The data used in this study are the fundamental mode of the Rayleigh waves recorded by the NARS stations during the ILIHA project [Paulssen,1990].
We have selected earthquakes for which the surface wavetrain has been recorded at a minimum of 4 stations. The frequency-band of the analysis is 10 to $60 \mathrm{mHz}$, but for most events the data are available in a more narrow band. This is due either to a decrease of signal over noise ratio inside the frequency band of interest, either to interference of different wavetrains which prohibit the measurement of a reliable phase at a number of frequencies. Among the earthquakes that were initially selected, the ones that gave very irregular phase velocities were rejected so that only the nine earthquakes in figure 1 have been used.

\section{Discussion of results}

With the phase data available we reconstructed wave fronts for the events listed in table 1, for frequencies between 10 and $50 \mathrm{mHz}$ and for frequencies up to $62 \mathrm{mhz}$ for the Azores event (nr.1). We tried both 2nd $(N=2, M=2)$ and 4 th $(\mathrm{N}=4, \mathrm{M}=4)$ order polynomials to adapt the phase function by iteration. For the number of points available (we had a maximum of 7 pairs of stations per event), not much difference is observed after both inversions, using different values of $\varepsilon$ in the range between 0.25 and 0.75 . The results are given for the 4th order polynomial inversion and for $\varepsilon=$ 0.5 . In figures 2 and 3 two examples are shown for events with a north-west (event $\mathrm{nr}$. 4) and north-east (event $\mathrm{nr}$. 2) back-azimuth respectively. All the events studied show a reasonable agreement between the theoretical and the experimental wave fronts, i.e. to a great extent it seems that waves arrive in the great circle direction. We should point out that changes on the starting velocity $c_{\text {in }}$ of about $0.1 \mathrm{~km} / \mathrm{s}$ gave variations of less than 2 degrees on the mean value of the angle. Two kinds of deviations from the shape of wave fronts in an homogeneous model are observed in the reconstruction of the wave fronts: a difference in the direction of arrival of the wave, and a difference in the curvature of the fronts. Waves crossing the Iberian Peninsula carry information both about the structure below Iberia and about the path followed before reaching this region. A difference in the direction of arrival indicates a deviation of the path of the true wave from the source-receiver minor arc, possibly due to refraction, scattering or/and multipathing effects before arriving to Iberia; a difference in curvature is an evidence of lateral heterogeneity both below Iberia and along the entire path.

On averaging the angles we have mixed these two effects. The value of the standard deviation can be helpful to distinguish one effect from the other: a relative high standard deviation implies a difference of curvature between the two kinds of fronts, which may correspond to any value of the average angle; a low standard deviation indicates that the curvature of the wave front is similar to the geometrical one,

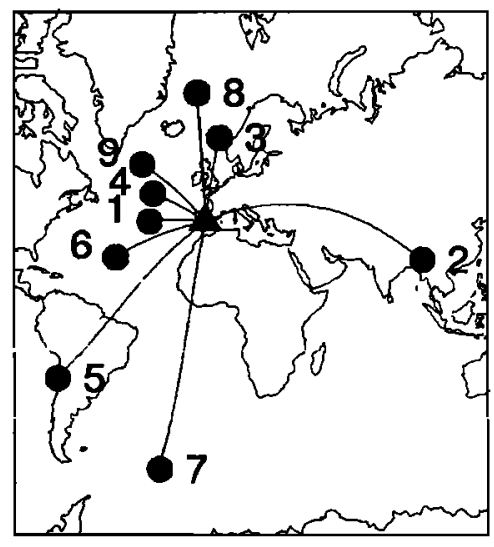

$\begin{array}{ccc}\mathrm{nr} & \text { date } & \mathrm{N} \\ 1 & 22-07-88 & 8 \\ 2 & 06-08-88 & 5 \\ 3 & 08-08-88 & 7 \\ 4 & 11-08-88 & 6 \\ 5 & 14-08-08 & 7 \\ 6 & 22-09-88 & 6 \\ 7 & 01-11-88 & 4 \\ 8 & 13-12-88 & 4 \\ 9 & 13-02-89 & 7\end{array}$

Fig. 1. Great Circle paths for the events used. Circles represent the epicenters; the triangle the location of the seismological network. $\mathrm{N}$ is the number of stations used. 


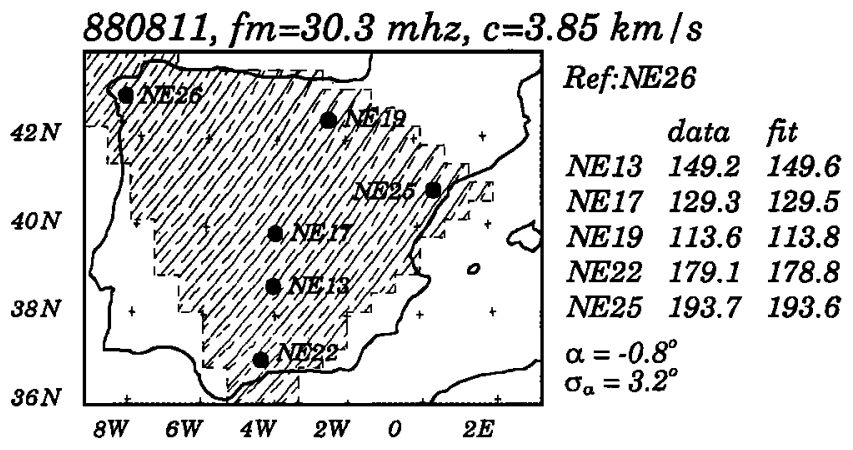

Fig. 2. Reconstruction of the wave fronts for the event 4, for a center frequency of $30.3 \mathrm{mHz}$. Solid lines give the wave fronts obtained from the inversion of the measured phase delays; dashed lines give the wave fronts corresponding to a homogeneous model with a reference phase velocity of 3.85 $\mathrm{km} / \mathrm{s}$. The inversion has only been performed in the region covered by the stations. The mean value for the angle in this region is $-0.8 \pm 3.2$ degrees. The circles represent the location of the stations used. Only the direction and the spacing of the lines are relevant, since they have been reconstructed using phase differences.

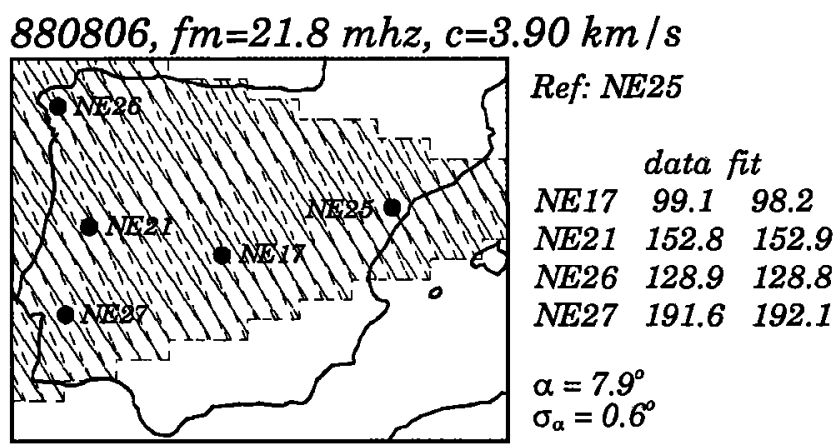

Fig. 3. Same as Figure 2 for the event 2.

and suggests a systematic deviation of the reconstructed wave fronts due to a different arrival direction. Figure 2 shows a typical example of wave front curvature difference, whereas figure 3 shows an example of a larger difference in direction of arrival. Figure 4 show the angles measured as a function of frequency. In figure 4(a) results for events with a pure oceanic great circle path are presented, whereas figure 4(b) shows the results for events with a great circle path partly oceanic and partly continental. Event 2 is the only event with a pure continental path.

For the events $1,4,6,9$ in figure 4(a) a difference in curvature is the dominant feature for all frequency bands, with values of the average angles much closer to zero than for the events in figure 4(b). In this case waves have been traveling along a relative short homogeneous oceanic path before reaching the Peninsula. This small deviation of the wave fronts from the great circle direction in the range of frequencies used, was already observed by Payo [1970] and Badal et al.[1990] and explained as an effect of the continental margin. For the event nr. 9, a general increase of the value of the angle and a decrease of the standard deviation with the frequency is observed. This means that for higher frequencies the deviation from the great circle path becames more important. This is what we in general would expect, since higher frequencies sample shallower depths with a more complex structure. However, this is not what we observe for all the events. For the Azores event (nr.1), for which a wider frequency band has been studied, the behaviour of the curve is quite irregular. This was already observed by Payo [1970] who indicated that these differences in the behaviour of the curves may be due to differences in the structure along seismic paths. For the events with a north and south backazimuth (events $3,7,8$ in figure 2), a difference in curvature is dominant for frequencies up to $30 \mathrm{mHz}$ whereas for higher frequencies a clear deviation from the great circle path is observed: in general higher angle values and smaller standard deviations are observed for higher frequencies. For these events the waves have crossed several continental margins to the North (North of Scotland and Bay of Biscay, events nr.3 and 8) and to the South (Coast of West Africa and Alboran Sea, event $\mathrm{nr} .7$ ). This last region is characterized by a complex lithospheric structure due to the active tectonic history [Paulssen,1990]. The fact that for some events the deviation increases at lower frequencies may come from less precise phase measurements. For the Chile event for which waves have traveled through the South American plate and along the Atlantic Ocean, an irregular pattern is observed: for frequencies around 25 and $35 \mathrm{mHz}$ it seems that there is a clear deviation from the great circle direction, with high values for the average angle and low standard deviation; for lower and higher frequencies a difference in curvature between the experimental and the theoretical wave fronts is observed. For the Thailand event (see figure 3 ) the reconstruction of the wave fronts suggests that the waves have traveled along another path than the great circle. This event gives the highest value (about 8 degrees) for frequencies between 10 and 25 $\mathrm{mHz}$. This event has complex waveforms; this is not surprising since the waves have traveled through complex continental structures; this notwithstanding it is surprising that simple wave fronts are obtained. Only frequencies up to $25 \mathrm{mHz}$ could be used for this event, due to the difficulty in obtaining reliable phase measurements for higher frequencies.

From the curves on figure 4 we see that the angles are small, up to 8 degrees, giving a relative error of less than $1 \%$ in the path-averaged value of the phase velocity:

$$
\varepsilon_{\mathrm{r}}=\frac{\left|\mathrm{c}_{\text {real }}-\mathrm{c}_{\text {meas }}\right|}{\mathrm{c}_{\text {real }}} \times 100 \%=\frac{1-\cos \alpha}{\cos \alpha} \times 100 \% \leq 1 \% \text { (7) }
$$

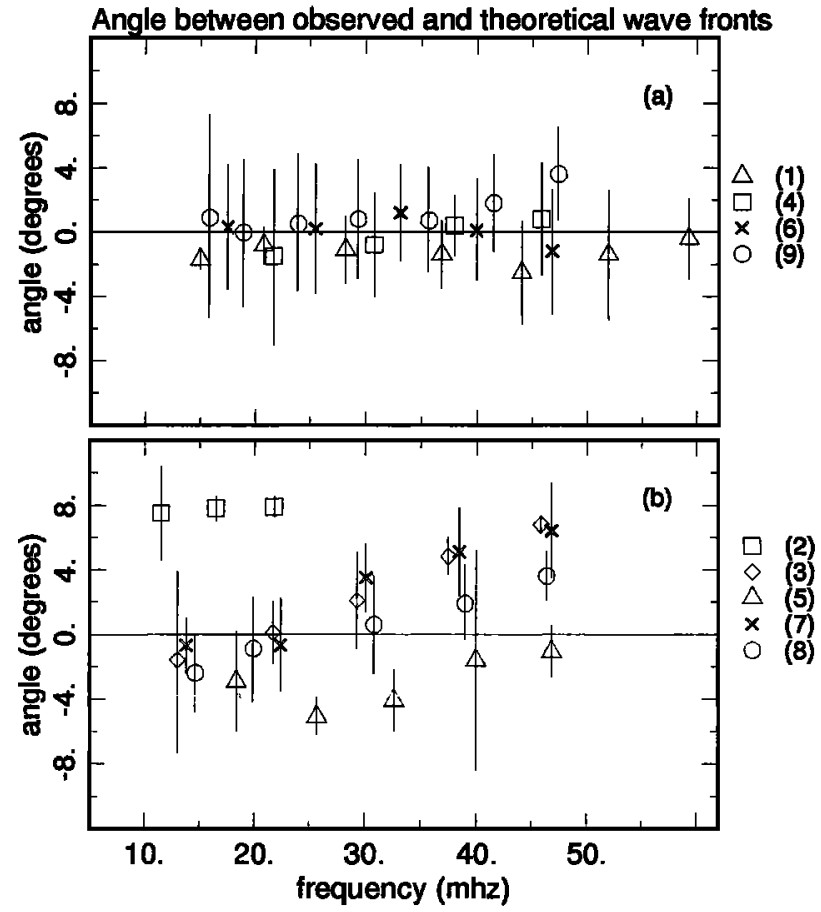

Fig. 4. Angles between wave fronts averaged in the region used in the inversion. Vertical bars indicate the standard deviation. In the legend the number refers to Figure 1. 
The observed deviation of the direction of the wave front can in principal be related to horizontal gradient of the phase velocity using the theory of Woodhouse and Wong [1986]. If the great circle approximation is valid, the direction of the observed wave fronts should match the polarization of the surface waves [Paulssen et al.,1990; Levshin et al.,1992]. The small relative error obtained implies that for the studied events and frequency bands, the great circle approximation can be used for dispersion measurements. However, the fact that a good agreement between the reconstructed wave fronts is achieved does not imply that the structures are smooth on a scale of the wavelength: one should not forget that because of the regularization in the equation (1), we select the solution that satisfies the Eikonal equation as good as possible, among all the ones that satisfy the data. As pointed out by Snieder [1988], S-velocity models reconstructed using surface wave scattering theory bear a close resemblance to models constructed largely based on surface wave dispersion measurements, with horizontal length scale comparable to the wavelength of the surface waves used, in which case scattering and multipathing may occur. Apparently, traditional methods for measuring phase velocities are rather robust to violations of the requirement that the heterogeneity is smooth on a scale of the wavelength. It seems that for a network of the size of ILIHA, the distance between stations is too small with respect to the wavelengths involved in this study, for the wave fronts to carry accurate information about the structure below Iberia. It follows that for the periods used we find smooth wave fronts satisfying the phase reasonably well, even though this doesn't mean that the structure is smooth. Having more wavelengths inside the network, i.e. working at higher frequencies, we would expect more complex wave fronts, giving more detailed information about the structure below Iberia. The problem is to find phase data at these frequencies; at periods smaller than 20s for oceanic paths the multipathing is very strong, and we found it very difficult to get results as method-independent as possible.

\section{Conclusions}

In this study it has been found that for surface waves traveling mostly through oceanic paths before reaching the region of study, and for frequencies between 10 and $60 \mathrm{mHz}$, a good agreement is achieved between the direction of arrival of the fundamental mode of the Rayleigh waves and the great circle. Angles up to 8 degrees have been obtained between the true and the theoretical wavefront, which represents a relative error of less than $1 \%$ in the path-averaged value of the phase velocity. This very good fit of the phase data with simple wave fronts (see figure 2 and 3 ) shows that the traditional methods for measuring phase velocities of surface waves are rather robust. One should not forget that few data have been used: only low frequencies for waves traveling mostly through an oceanic path before reaching the Iberian Peninsula.
Acknowledgments. This research was supported by the Spanish Ministry of Education and Science through a $\mathrm{PhD}$ grant (FPI), and by the Netherlands Organization for Scientific Research (NWO) through the Pionier project PGS 76-144. The EEC is gratefully acknowledged for their financial support to the ILIHA project.

\section{References}

Badal, J., V. Corchete, G. Payo, J. A. Canas, L. Pujades and F. J. Serón, Processing and inversion of long-period surface-wave data collected in the Iberian Peninsula, Geophys.JInt., 100, 193-202, 1990.

Dziewonski, A. M., J. Mills and S. Block, Residual dispersion measurement-a new method of surface-wave analysis, Bull. seism. Soc. Am., 62, 129-139, 1972.

Evernden, J. F., Direction of approach of Rayleigh waves and related problems (Part II), Bull. seism. Soc. Am., 44, 159-184, 1954.

Knopoff, L., M. J. Berry and F. Schwab, Tripartite phase velocity observations in laterally heterogeneous regions, $J$. geophys. Res., 72, 2595-2601, 1967.

Levshin, A. L., L. I. Ratnikova and J. Berger, Peculiarities of surface-wave propagation across central Eurasia, Bull. seism. Soc. Am., 82, 2464-2493, 1992.

Paulssen, H., The Iberian Peninsula and the ILIHA Project, Terra Nova, 2, 429-435, 1990.

Paulssen, H., A. L. Levshin, A. V. Lander and R. Snieder, Time- and frequency-dependent polarization analysis: anomalous surface waves observations in Iberia, Geophys.J.Int., 103, 483-496, 1990.

Payo, G., Structure of the Crust and Upper Mantle in the Iberian Shield by means of a Long Period Triangular Array, Geophys.J.R.astr.Soc., 20, 493-508, 1970.

Schwab, F. and E. Kausel, Quadripartite Surface Wave Method: Development, Geophys. J. R. astr. Soc, 45, 231-244, 1976.

Snieder, R., Large-Scale Waveform Inversions of Surface Waves for Lateral Heterogeneity, 2, Application to Surface Waves in Europe and the Mediterranean, J. geophys. Res., 93, 12,067-12,080, 1988.

Woodhouse, J. H. and Y. K. Wong, Amplitude, phase and path anomalies of mantle waves, Geophys.J.R.astr.Soc., 87, 753-774, 1986.

$\overline{D .}$ Alsina and R. Snieder, Department of Theoretical Geophysics, Institute of Earth Sciences, Utrecht University, P.O. Box 80.021, 3508 TA Utrecht, The Netherlands

V. Maupin, Institut de Physique du Globe de Strasbourg, 5 rue Descartes, 67084 Strasbourg Cedex, France.

(Received: January 25, 1993; revised: March 22, 1993; accepted: March 22, 1993.) 